\title{
D Vitamin D Increases CTLA-4 Gene Expression in Patients with Mild to Moderate Ulcerative Colitis
}

\author{
Amrollah Sharifi ${ }^{1}$, Homayoon Vahedi ${ }^{2, *}$, Mohammad Reza Honarvar ${ }^{3}$, Behnam Alipoor ${ }^{4}$, Zeinab Nikniaz ${ }^{5}$, \\ Hossein Rafiei ${ }^{6}$, Mohammad Javad Hosseinzadeh-Attar ${ }^{7}$
}

1. Assistant Professor; Golestan Research Center of Gastroenterology and Hepatology (GRCGH), Faculty of health, Golestan University of Medical Sciences (GOUMS), Gorgan, Iran

2. Associate Professor; Digestive Disease Research Center, Digestive Research Institute, Shariati Hospital, Tehran University of Medical Sciences, Tehran, Iran

3. Assistant Professor, Health Management and Social Development Research Center, Faculty of Health, Golestan University of Medical Sciences, Gorgan, Iran

4. Assistant Professor; Department of Laboratory Sciences, Faculty of Paramedicine, Yasuj University of Medical Sciences, Yasuj, Iran

5. Assistant Professor; Gastrointestinal Diseases Research Center, Tabriz University of Medical Sciences, Tabriz, Iran.

6. Faculty of Health and Social Development, College of Health and Exercise Sciences, University of British Columbia Okanagan, Kelowna, BC, Canada

7. Professor; Department of Clinical Nutrition, School of Nutritional Sciences and Dietetics, Tehran University of Medical Sciences, Tehran, Iran

* Corresponding Author:

Homayoon Vahedi, MD

North Kargar St, Dr Shariati Hospital, Digestive Disease Research Institute,

Tehran, Iran

Tel: + 982182415104

Fax: +982182415400

Email: homayoonvahedi@gmail.com

Received: 17 May. 2019

Accepted: 14 Aug. 2019

\section{ABSTRACT}

\section{BACKGROUND}

Ulcerative colitis (UC) is a chronic inflammatory disorder of the large intestine. Cytotoxic T-lymphocyte-associated protein 4 (CTLA-4) is a member of the immunoglobulin superfamily, which binds B7-1 and B7-2 on APCs (antigen-presenting cells), and induces APCs to produce an inhibitory signal to $\mathrm{T}$ cells. The aim of this study was to investigate the effect of vitamin $\mathrm{D}$ on CTLA-4 gene expression in whole blood samples of patients with UC.

\section{METHODS}

90 patients with mild to moderate $\mathrm{UC}$ were randomized to receive either a single injection of $7.5 \mathrm{mg}$ vitamin D3 or $1 \mathrm{~mL}$ normal saline. 90 days following the intervention fold changes in CTLA-4 mRNA expression were determined and statistical comparisons between the two groups were performed.

\section{RESULTS}

Serum vitamin D increased significantly only in the vitamin D group. CTLA-4 fold changes were significantly higher in the vitamin $\mathrm{D}$ group compared with the placebo group (median $\pm \mathrm{IQR}$ : $1.21 \pm 2.3$ vs. $1.00 \pm 1.5$, respectively; $p=0.007)$.

\section{CONCLUSION}

The results of this study revealed that vitamin D administration in patients with UC enhances the CTLA-4 gene expression.

\section{KEYWORDS:}

Ulcerative Colitis; Inflammatory Bowel Disease; CTLA-4; Vitamin D; Gene Expression

\section{Please cite this paper as:}

Sharifi A, Vahedi H, Honarvar MR, Alipoor B, Nikniaz Z, Rafiei H, Hosseinzadeh-Attar MJ. Vitamin D Increases CTLA-4 Gene Expression in Patients with Mild to Moderate Ulcerative Colitis. Middle East J Dig Dis 2019;11:199-204. doi:10.15171/mejdd.2019.149.

\section{INTRODUCTION}

Inflammatory bowel disease (IBD) is a chronic relapsing-remitting inflammatory disorder of the alimentary tract, which includes two main forms, ulcerative colitis (UC) and Crohn's disease (CD). Although to date the etiology of IBD has not been completely understood, a body of evidence suggests that genetic abnormalities coupled with particular environmental factors may lead to IBD. ${ }^{1-5}$ In this regard different specific genetic abnormalities have been identified, which are responsible for IBD. One of them is genetic variants of the cytotoxic T-

口ifa (C) 2019 The Author(s). This work is published by Middle East Journal of Digestive Diseaes as an open access article distributed under the terms of the Creative Commons Attribution License (https://creativecommons. 
lymphocyte-associated protein ${ }^{4}$ (CTLA-4) that has been shown to be related to various autoimmune diseases, ${ }^{6-10}$ including IBD. ${ }^{11}$ A recent meta-analysis suggested that the CTLA-4 + 49A/G variant may be associated with susceptibility to CD, and the CTLA-4 CT60 variant may increase the risk of UC in Asians. ${ }^{12}$

CTLA-4, also known as CD125 (cluster of differentiation 152), is mainly expressed on the surface of T lymphocytes, functioning as an immune checkpoint, which produces an inhibitory signal to T cells. ${ }^{10}$ CTLA4 and CD28 have a sequence homology, and both can attach to B7-1 and B7-2 (CD80 and CD86) on the APCs with different affinities. CTLA-4 binds B7-1 and B7-2 with higher affinity, and as a negative regulator in immune system induces an inhibitory signal to T cells, whereas binding of CD28 to B7-1 and B7-2 induces a stimulatory signal. ${ }^{13-15}$ The comparatively higher binding affinity of CTLA-4 to B-7 receptors has made it a potential and promising candidate for attenuating immune responses in autoimmune diseases. So, in this regard, previous research has focused on factors that increase the expression of CTLA-4 in inflammatory diseases like rheumatoid arthritis and showed its promising effect in increasing remission in such patients.

Vitamin D has been shown to antagonize the inhibitory effect of inflammatory cytokines on CTLA-4 and enhances CTLA4-induced suppression of inflammation. ${ }^{16}$ In addition, calcitriol increased CTLA-4 gene expression in human CD4+ CD25- T-cell cultures. ${ }^{17}$ It also increased CTLA4 expression in $\mathrm{T}$ cells of patients with type 1 diabetes mellitus with a CD4 + CD25 high and CD127 low profile. ${ }^{18}$ Furthermore, vitamin D enhanced the CTLA-4 expression in both healthy and patients with latent tuberculosis (TB) ${ }^{19}$ Therefore, it seems that calcitriol (the active form of vitamin D) has a synergistic effect with CTLA-4 in the suppression of inflammatory responses. In addition, in vitro studies revealed that calcitriol enhanced the expression of CTLA-4 by direct effects upon the T cell even in the presence of Th17 polarizing cytokines. ${ }^{16}$ However, the possible direct effect of vitamin D on CTLA-4 expression has not yet been clarified in vivo.

Considering the anti-inflammatory effects of vitamin $\mathrm{D}$ in patients with $\mathrm{UC},{ }^{20}$ and its effect on increasing the CTLA-4 expression in in vitro studies, ${ }^{16}$ we hypothesized that the anti-inflammatory effects of vitamin $\mathrm{D}$ in patients with IBD may be partly related to its ability to increase
CTLA-4 expression. Therefore, in the present clinical trial for the first time, we investigated vitamin D effect on CTLA-4 gene expression in whole blood samples of patients with mild to moderate UC.

\section{MATERIALS AND METHODS}

In this two-arm double-blind randomized placebocontrolled trial, following primary assessment for eligibility, 90 patients with UC were randomized to receive either a single injection of $7.5 \mathrm{mg}$ vitamin D3 (vitamin D group) or $1 \mathrm{~mL}$ normal saline (placebo group). ${ }^{20}$ Investigators and patients were kept masked to group assignments, and allocation/injection staff did not participate in any of the study clinical evaluations or assays. The complete details of the inclusion and exclusion criteria and characteristics of the patients have been discussed before. ${ }^{20}$ The study was conducted using the 90 cDNA samples of our previous study, and at the significant level of less than $5 \%$, the precision power higher than $80 \%$ was obtained.

The trial was registered at Iranian Registry of Clinical Trials (IRCT) (register number: IRCT2014062318207N1). The study was approved by the Ethics Committee of Golestan University of Medical Sciences. Written informed consent was obtained from all the patients prior to participating in the study.

\section{Experiments:}

Before (maximum 30 minutes) and 3 months after the intervention, fasting blood samples were collected. Total RNA was immediately isolated from whole blood using QIAamp RNA Blood Mini Kit (Qiagen, USA), and treated with DNase 1 . The optical density of each RNA isolate was measured at 280,260, and $230 \mathrm{~nm}$ to control for quality and quantity of RNA. Reverse transcription of 1 $\mu \mathrm{g}$ RNA was performed using GeneAll HyperScriptTM Reverse Transcriptase (GeneAll, South Korea) with oligo-dT and random hexamer primers in $20 \mu \mathrm{L}$ reaction volume and cDNA of all samples were obtained. The cDNA samples were stored at -80 degrees Celsius until the real-time polymerase chain reaction (PCR) tests. Duplicate real-time quantitative reverse transcriptase PCR (qRT-PCR) were performed by the LightCycler ${ }^{\circledR} 2.0$ Instrument (Roche, Germany) for determination of CTLA4 relative gene expression. Succinate dehydrogenase complex subunit A (SDHA) gene was used as a reliable 


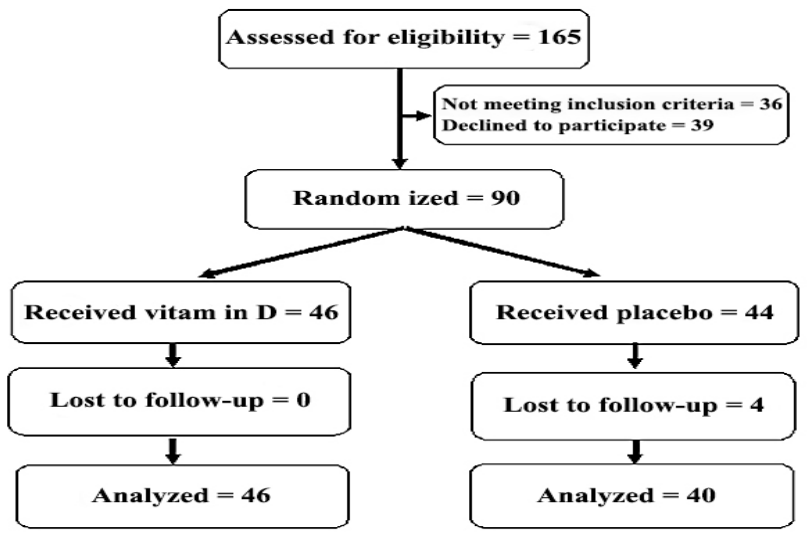

Fig.1: Consort flow diagram of the study.

internal control. ${ }^{21}$ PCR was run in a final reaction volume of $20 \mu \mathrm{L}$, containing $10 \mu \mathrm{L}$ of $2 \times$ SYBR Green Master Mix (Ampliqon; Denmark), $1 \mu \mathrm{L}$ (10 ng) of forward and reverse primers, and $1 \mu \mathrm{L}$ of cDNA (equivalent to about 50 ng RNA) and PCR-grade water. Reaction mixtures either without reverse transcriptase or template (NTC) were included in each experiment for quality control. The standard curves for both CTLA-4 and SDHA of four serial dilutions of cDNA (50 ng to $50 \mathrm{pg}$ ) were plotted. All PCR efficiencies were above 95\%.

The CTLA-4 (NM_001037631) and SDHA (NM_001294332) primers were designed by Primer3 software. Online medical databases were also searched for obtained primers to find out if they have been used before. Primers for CTLA-4 were as follow: forward: 5'- CTACCTGGGCATAGGCAACG -3', reverse: 5'CCCCGAACTAACTGCTGCAA -3', and for SDHA: forward: 5'- TGGGAACAAGAGGGCATCTG-3', reverse: 5'- CCACCACTGCATCAAATTCATG-3. ${ }^{22}$

The amplification profile was as follows: 10 minutes initial denaturation at $95^{\circ} \mathrm{C}$ followed by 40 cycles of 15 seconds denaturation $\left(95^{\circ} \mathrm{C}\right), 20$ seconds annealing $\left(56^{\circ} \mathrm{C}\right)$, and 20 seconds extensions $\left(72^{\circ} \mathrm{C}\right)$. At the end of each run, PCR machine was set for 15 seconds at $95^{\circ} \mathrm{C}$, 60 seconds at $60^{\circ} \mathrm{C}$, and the final temperature at $95^{\circ} \mathrm{C}$ with $0.1{ }^{\circ} \mathrm{C} / \mathrm{s}$ slope, to plot the melting curve.

Fold changes in CTLA-4 mRNA expression normalized to SDHA and relative to baseline values were determined for each patient using the $2^{-\Delta \Delta \mathrm{Cq}}$ method ${ }^{23}$ by a Microsoft Excel file (Microsoft Office 2016) formulated specifically for this study, on which $\Delta \Delta \mathrm{Cq}=\left(\mathrm{Cq}_{\text {CTLA- }}\right.$ 4 After $\left.-\mathrm{Cq}_{\text {SDHA After }}\right)-\left(\mathrm{Cq}_{\text {CTLA-4 Before }}-\mathrm{Cq}_{\text {SDHA Before }}\right)$.

\section{Statistical analysis:}

Statistical analyses were done by SPSS software version 25.0 (IBM SPSS Inc., Chicago, Illinois, United States). The normality of distribution was checked using Kolmogorov-Smirnov test. Skewed distribution data were expressed as medians \pm interquartile ranges. Comparisons between the two groups were performed using independent $t$ test for variables with normal distribution and Mann-Whitney $U$ test for variables with skewed distribution. Spearman's rho test was used for the correlation analyses. Statistically significant level was set at $p$ value $<0.05$.

\section{RESULTS}

86 patients (40 in the placebo group, and 46 in the vitamin D group) completed the study. Four in the placebo group refused to complete the study. The CONSORT flow diagram of the trial is presented in figure 1.

At baseline, mean serum levels of $25(\mathrm{OH}) \mathrm{D}_{3}$ of vitamin $\mathrm{D}$ and placebo groups were $33.3 \pm 7.0$ and 32.9 $\pm 9.6 \mathrm{ng} / \mathrm{mL}$, respectively $(p=0.82)$ and 90 days after the intervention, serum vitamin D levels were higher compared with the baseline levels only in the vitamin D group, which were also significantly higher than the placebo group $(40.8 \pm 5.2$ and $33.9 \pm 10.6$, respectively, $p<0.001) .{ }^{20,24}$ The baseline values of serum calcium, parathormone, hs-CRP, erythrocyte sedimentation rate (ESR), dietary vitamin D, calcium intake, calorie intake and macronutrient composition, age, disease duration, systolic and diastolic blood pressure, body mass index (BMI), heart rate, and body temperature were not statistically different between the two groups. Drug regimen and sex proportion were also comparable. ${ }^{20,24}$

According to the result of Mann-Whitney $U$ test, CTLA-4 gene expression fold changes were significantly higher in the vitamin D group compared with the placebo group (median $\pm \mathrm{IQR}: 1.21 \pm 2.3$ vs. $1.00 \pm 1.5$, respectively; $p=0.007)$.

We also did a subgroup analysis regarding the baseline vitamin D levels (lower, equal, or higher than $30 \mathrm{ng} /$ $\mathrm{mL}$ ). Gene expression fold change was only significantly higher in the vitamin D group only in whom with baseline vitamin D levels equal to and higher than $30 \mathrm{ng} /$ $\mathrm{mL}(p=0.029$, figure 2$)$.

There was a negative but not significant correlation 


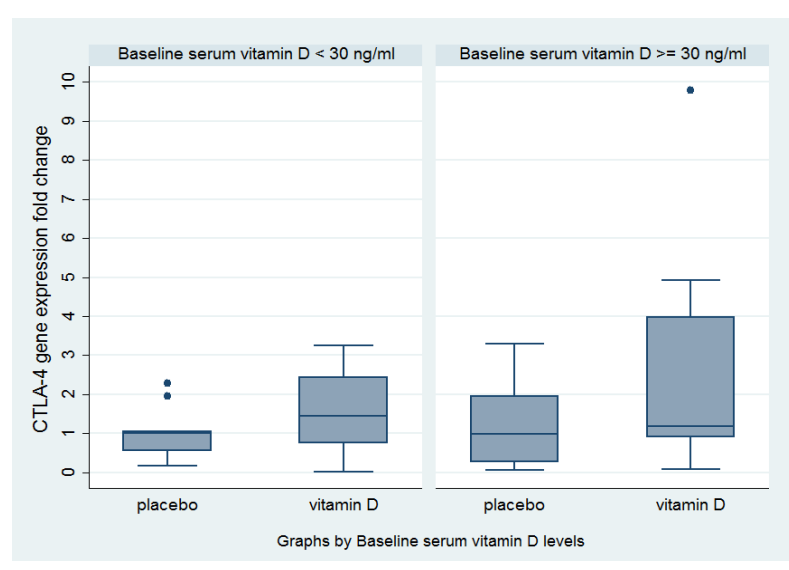

Fig.2: fold change in CTLA-4 (Cytotoxic T lymphocyte associated 4) gene expression normalized to SDHA (Succinate dehydrogenase complex subunit A) and calibrated for baselines regarding baseline vitamin D levels (median \pm IQR).

between CTLA-4 gene expression fold change and hsCRP levels (Spearman's rho $=-0.2 ; p=0.056$ ). In addition, there was a significant negative correlation between CTLA-4 gene expression fold change and ESR levels (Spearman's rho $=-0.31 ; p=0.004$ ).

\section{DISCUSSION}

We have previously shown that vitamin D supplementation in patients with UC could have a beneficial antiinflammatory effect, ${ }^{20}$ however, its underlying mechanisms remained unclear. One proposed mechanism, through which this may occur, is its effect on immune related gene expressions such as CTLA-4. Previously, an in vitro study showed that calcitriol enhanced the expression of CTLA-4 by direct effects on the T cell even in the presence of Th17 polarizing cytokines. ${ }^{16}$ This effect has also been shown by other studies. ${ }^{17-19}$ To the best of our knowledge, there is no clinical trial evidence for this effect. The results of our study for the first time revealed that vitamin $\mathrm{D}$ administration in patients with mild to moderate UC enhances the CTLA-4 gene expression. A significant negative correlation between CTLA-4 gene expression and ESR levels (as a clinical inflammatory marker) was also found.

Altering the expression of CTLA-4 gene is an important pathway by which T-reg cells and other CTLA4 expressing immune cells evoke immune tolerance. ${ }^{25}$ The expression of CTLA-4 by effector T cells has been shown to affect the $\mathrm{T}$ cell response duration. ${ }^{26,27}$ which may be a crucial part of $\mathrm{T}$ cell anergy after the activation of immune system. These findings highlight the importance of suppressing immune responses in the treatment of autoimmune diseases such as IBD, in which induction of CTLA-4, for example, might decrease the inflammation in such pathological situations.

It is now demonstrated that peripheral monocytes, leukocytes, and activated CD4 + and CD8 + T cells possess vitamin D receptor (VDR). ${ }^{28}$ Furthermore, $1,25(\mathrm{OH})_{2} \mathrm{D}_{3}$ was shown to decrease the proliferation of Th1 and Th17 cells while stimulation of T regulatory cell, ${ }^{29}$ was supported by the evidence of the link between vitamin $\mathrm{D}$ deficiency with the severity of IBD, ${ }^{30}$ and supported by the idea that vitamin $\mathrm{D}$ administration could produce beneficial anti-inflammatory impacts in patients with UC. ${ }^{20}$ It has been proposed that the principal way by which vitamin $\mathrm{D}$ diminishes $\mathrm{T}$ cell responses is via down-regulation of co-stimulatory molecules on APCs.${ }^{31}$ Accordingly, in vitro studies have shown that calcitriol induces CTLA-4 through direct effects on the T cells. Moreover, the suppressive effect of calcitriol on $\mathrm{T}$ cells is further boosted through the removal of CD80 and CD86 by CTLA4-mediated transendocytosis. ${ }^{16}$ All these findings suggest that low levels of vitamin D followed by decreased CTLA-4 expression could lead to exaggerated inflammatory reactions in pathological inflammatory situations, ${ }^{16}$ the hypothesis that has now been shown in our study.

The results of this study also showed that vitamin D supplementation had significant effect on CTLA-4 gene expression in whom with baseline sufficient vitamin D levels, by which it can be concluded that for achieving an efficient effect on CTLA-4 gene expression, final vitamin $\mathrm{D}$ levels higher than the current common normal level cut-offs might be needed. However, in this regard low statistical power of subgroup analysis must be considered.

In conclusion in the current study, we showed that vitamin D supplementation in patients with UC upregulates CTLA-4 gene expression as a protective mechanism against the severity of the disease. As we have noted before ${ }^{20,24}$ there were some limitations in this study. First of all, we did not screen patients for baseline vitamin D deficiency. Second, only patients with mild to moderate UC were included. Third, we did not assess the dose-response effect of vitamin D. Therefore, larger studies with multiple doses of vitamin $\mathrm{D}$ and longer observations 
periods are encouraged. Conducting a similar study in vitamin D deficient patients with $\mathrm{UC}$ in relapse phase using different doses of vitamin $\mathrm{D}$ is suggested.

\section{ACKNOWLEDGMENTS:}

This work was supported by Golestan University of Medical Sciences and Health Services (grant No. 970121003).

\section{Authors' contributions:}

Amrollah Sharifi: Literature search; the conception and design of the study; carry out the study, acquisition, statistical analysis, and interpretation of the data; drafting the manuscript; final approval of the version to be submitted; agreement to be accountable for all aspects of the work.

Homayoon Vahedi: Design of the study; interpretation of data; revising the manuscript critically for important intellectual content; final approval of the version to be submitted; clinical supervision;. agreement to be accountable for all aspects of the work.

Mohammad Reza Honarvar: Acquisition of data, revising the manuscript critically for important intellectual content, final approval of the version to be submitted; agreement to be accountable for all aspects of the work.

Behnam Alipoor: Acquisition of data; interpretation of data; revising the manuscript critically for important intellectual content; final approval of the version to be submitted;. agreement to be accountable for all aspects of the work.

Zeinab Nikniaz: Acquisition of data; interpretation of data; revising the manuscript critically for important intellectual content; final approval of the version to be submitted;. agreement to be accountable for all aspects of the work.

Hossein Rafiei: Acquisition of data; interpretation of data; revising the manuscript critically for important intellectual content; final approval of the version to be submitted;. agreement to be accountable for all aspects of the work.

Mohammad Javad Hosseinzadeh-Attar: Design of the study; interpretation of data; revising the manuscript critically for important intellectual content; final approval of the version to be submitted;. agreement to be accountable for all aspects of the work.

\section{ETHICAL APPROVAL}

There is nothing to be declared.

\section{CONFLICT OF INTEREST}

The authors declare no conflict of interest related to this work.

\section{REFERENCES}

1. Zhang YZ, Li YY. Inflammatory bowel disease: pathogenesis. World $J$ Gastroenterol 2014;20:91-9. doi: 10.3748/wjg.v20.i1.91.

2. Khor B, Gardet A, Xavier RJ. Genetics and pathogenesis of inflammatory bowel disease. Nature 2011;474:307-17. doi: 10.1038/nature10209.

3. Orholm M, Binder V, Sorensen TI, Rasmussen LP, Kyvik KO. Concordance of inflammatory bowel disease among Danish twins. Results of a nationwide study. Scand J Gastroenterol 2000;35:1075-81. doi: 10.1080/003655200451207.

4. Spehlmann ME, Begun AZ, Burghardt J, Lepage P, Raedler A, Schreiber S. Epidemiology of inflammatory bowel disease in a German twin cohort: results of a nationwide study. Inflamm Bowel Dis 2008;14:968-76. doi: 10.1002/ibd.20380.

5. Halfvarson J. Genetics in twins with Crohn's disease: less pronounced than previously believed? Inflamm Bowel Dis 2011;17:6-12. doi: 10.1002/ibd.21295.

6. Kuehn HS, Ouyang W, Lo B, Deenick EK, Niemela JE, Avery DT, et al. Immune dysregulation in human subjects with heterozygous germline mutations in CTLA4. Science 2014;345:1623-7. doi: 10.1126/science.1255904.

7. Tang MJ, Zhou ZB. Association of the CTLA-4 +49A/G polymorphism with rheumatoid arthritis in Chinese Han population. Mol Biol Rep 2013;40:2627-31. doi: 10.1007/ s11033-012-2349-6.

8. Ahmed S, Ihara K, Kanemitsu S, Nakashima H, Otsuka T, Tsuzaka K, et al. Association of CTLA-4 but not CD28 gene polymorphisms with systemic lupus erythematosus in the Japanese population. Rheumatology (Oxford) 2001;40:662-7. doi: 10.1093/rheumatology/40.6.662.

9. Kouki T, Sawai Y, Gardine CA, Fisfalen ME, Alegre ML, DeGroot LJ. CTLA-4 gene polymorphism at position 49 in exon 1 reduces the inhibitory function of CTLA-4 and contributes to the pathogenesis of Graves' disease. $J$ Immunol 2000;165:6606-11. doi: 10.4049/jimmunol.165.11.6606.

10. Wang K, Zhu Q, Lu Y, Lu H, Zhang F, Wang X, et al. CTLA-4 +49 G/A Polymorphism Confers Autoimmune Disease Risk: An Updated Meta-Analysis. Genet Test Mol Biomarkers 2017;21:222-7. doi: 10.1089/ gtmb.2016.0335

11. Zeissig S, Petersen BS, Tomczak M, Melum E, HucClaustre E, Dougan SK, et al. Early-onset Crohn's disease 
and autoimmunity associated with a variant in CTLA-4. Gut 2015;64:1889-97. doi: 10.1136/gutjnl-2014-308541.

12. Zhang M, Ni J, Xu WD, Wen PF, Qiu LJ, Wang XS, et al. Association of CTLA-4 variants with susceptibility to inflammatory bowel disease: a meta-analysis. Hum Immunol 2014;75:227-33. doi: 10.1016/j.humimm.2013.12.008.

13. Felix NJ, Suri A, Salter-Cid L, Nadler SG, Gujrathi S, Corbo $\mathrm{M}$, et al. Targeting lymphocyte co-stimulation: from bench to bedside. Autoimmunity 2010;43:514-25. doi: 10.3109/08916931003674741.

14. Walker LS, Sansom DM. Confusing signals: recent progress in CTLA-4 biology. Trends Immunol 2015;36:63-70. doi: 10.1016/j.it.2014.12.001

15. Kim G, Schoenberger SP, Sharpe A, Kronenberg M. Synergistic costimulation by both B7 molecules regulates colitis pathogenesis. Ann N Y Acad Sci 2006;1072:23341. doi: 10.1196/annals.1326.012.

16. Jeffery LE, Qureshi OS, Gardner D, Hou TZ, Briggs Z, Soskic B, et al. Vitamin D Antagonises the Suppressive Effect of Inflammatory Cytokines on CTLA-4 Expression and Regulatory Function. PloS One 2015;10:e0131539. doi: 10.1371/journal.pone.0131539.

17. Jeffery LE, Burke F, Mura M, Zheng Y, Qureshi OS, Hewison M, et al. 1,25-Dihydroxyvitamin D3 and IL-2 combine to inhibit $T$ cell production of inflammatory cytokines and promote development of regulatory T cells expressing CTLA-4 and FoxP3. J Immunol 2009;183:5458-67. doi: 10.4049/jimmunol.0803217.

18. Van Belle TL, Vanherwegen AS, Feyaerts D, De Clercq P, Verstuyf A, Korf H, et al. 1,25-Dihydroxyvitamin D3 and its analog TX527 promote a stable regulatory T cell phenotype in T cells from type 1 diabetes patients. PloS One 2014;9:e109194. doi: 10.1371/journal.pone.0109194.

19. Hawthorne G, JinJin Z, Thickett D, Turner A. Vitamin $\mathrm{D}$ supplementation in health and latent tuberculosis promotes regulatory T cell expression. European Respir $J$ 2016;48(suppl 60):PA2116. doi: 10.1183/13993003.

20. Sharifi A, Hosseinzadeh-Attar MJ, Vahedi H, Nedjat S. A randomized controlled trial on the effect of vitamin D3 on inflammation and cathelicidin gene expression in ulcerative colitis patients. Saudi J Gastroenterol 2016;22:316-23. doi: 10.4103/1319-3767.187606.

21. Ledderose C, Heyn J, Limbeck E, Kreth S. Selection of reliable reference genes for quantitative real-time PCR in human T cells and neutrophils. BMC Res Notes 2011;4:427. doi: 10.1186/1756-0500-4-427.

22. Pereira F, Barbachano A, Singh PK, Campbell MJ, Munoz A, Larriba MJ. Vitamin D has wide regulatory effects on histone demethylase genes. Cell Cycle 2012;11:1081-9. doi: 10.4161/cc.11.6.19508.

23. Livak KJ, Schmittgen TD. Analysis of relative gene expression data using real-time quantitative PCR and the 2(-Delta Delta C(T)) Method. Methods 2001;25:402-8. doi: 10.1006/meth.2001.1262.

24. Read S, Malmstrom V, Powrie F. Cytotoxic T lymphocyte- associated antigen 4 plays an essential role in the function of $\mathrm{CD} 25(+) \mathrm{CD} 4(+)$ regulatory cells that control intestinal inflammation. $J$ Exp Med 2000;192:295-302. doi: 10.1084/jem.192.2.295.

25. Wang CJ, Kenefeck R, Wardzinski L, Attridge K, Manzotti C, Schmidt EM, et al. Cutting edge: cell-extrinsic immune regulation by CTLA-4 expressed on conventional T cells. J Immunol 2012;189:1118-22. doi: 10.4049/jimmunol.1200972.

26. Corse E, Allison JP. Cutting edge: CTLA-4 on effector T cells inhibits in trans. J Immunol 2012;189:1123-7. doi: 10.4049/jimmunol.1200695.

27. Lim WC, Hanauer SB, Li YC. Mechanisms of disease: vitamin D and inflammatory bowel disease. Nat Clin Pract Gastroenterol Hepatol 2005;2:308-15. doi: 10.1038/ncpgasthep0215.

28. Cantorna MT. Why do T cells express the vitamin D receptor? Ann N Y Acad Sci 2011;1217:77-82. doi: 10.1111/j.1749-6632.2010.05823.x.

29. Ye L, Lin Z, Liu J, Cao Q. Vitamin D Deficiency Is Associated with Endoscopic Severity in Patients with Crohn's Disease. Gastroenterol Res Pract 2017;2017:4869718. doi: 10.1155/2017/4869718.

30. Penna G, Adorini L. 1 Alpha,25-dihydroxyvitamin D3 inhibits differentiation, maturation, activation, and survival of dendritic cells leading to impaired alloreactive $\mathrm{T}$ cell activation. J Immunol 2000;164:2405-11. doi: 10.4049/ jimmunol.164.5.2405.

31. Fawaz L, Mrad MF, Kazan JM, Sayegh S, Akika R, Khoury SJ. Comparative effect of $25(\mathrm{OH})$ D3 and 1, $25(\mathrm{OH}) 2 \mathrm{D} 3$ on Th17 cell differentiation. Clin Immunol 2016;166:59-71. doi: 10.1016/j.clim.2016.02.011. 